\title{
Métodos científicos de indagación y de construcción del conocimiento
}

Andrés Rodríguez Jiménez ${ }^{1}$ Universidad de Artemisa andresrj1955@gmail.com

Alipio Omar Pérez Jacinto² Universidad de Artemisa opejota@gmail.com

DOI: https://doi.org/10.21158/01208160.n82.2017.1647

Fecha de recepción: 21 de diciembre de 2016

Fecha de aprobación: 1 de marzo de 2017

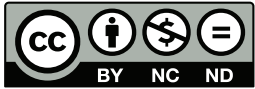

Cómo citar este artículo/ To reference this article/ Comment citer cet article/ Para citar este artigo: Rodríguez, A. y Pérez, A. O. (2017). Métodos científicos de indagación y de construcción del conocimiento Revista EAN, 82, pp.175-195. https://doi.org/10.21158/01208160.n82.2017.1647

\section{Resumen}

Los métodos de investigación que se describen en la literatura son una importante herramienta para la búsqueda y el perfeccionamiento del conocimiento acerca de la realidad. Cada método tiene su forma particular de acercamiento al objeto de estudio, lo cual origina diferentes clasificaciones. El siguiente trabajo pretende fundamentar una nueva perspectiva de clasificación de estos métodos, de acuerdo con su función en el proceso de investigación. Se ha realizado una sistematización de referentes bibliográficos que ha posibilitado caracterizar los métodos más comúnmente empleados y clasificarlos según su finalidad investigativa, aspecto que resulta novedoso e importante para la metodología de investigación. La propuesta de una distinción entre los métodos que privilegian la búsqueda de información de aquellos que se enfocan en la construcción de conocimiento, resulta de vital importancia para el investigador, quien tiene la posibilidad de utilizar dicha información como una herramienta de orientación al momento de seleccionar el método más pertinente según el objetivo de su proyecto.

\section{Palabras clave}

Métodos de investigación, conocimiento científico, métodos teóricos, métodos racionales, métodos empíricos, conocimiento empírico, conocimiento racional.

\footnotetext{
${ }^{1}$ Licenciado en Educación con especialidad en Química por la Universidad de Ciencias Pedagógicas Enrique J. Varona de La Habana. Magíster en Ciencias de la Educación Superior por la Universidad de La Habana. Doctor en Ciencias Pedagógicas por la Universidad de La Habana. Director del Centro de Estudios de Educación y Desarrollo de la Universidad de Artemisa de Cuba.

${ }^{2}$ Licenciado en Física por la Universidad de La Habana. Magíster en Ciencias de la Educación por la Universidad de Ciencias Pedagógicas Enrique J. Varona de La Habana). Doctor en Ciencias Pedagógicas (Universidad de Ciencias Pedagógicas Enrique J. Varona de La Habana. Metodólogo del Vicerrectoría de Desarrollo y Tecnología de la Universidad de Artemisa de Cuba.
} 


\title{
Scientific methods of surveying and building knowledge
}

\begin{abstract}
Research methods described in contemporary literature become significant tools to search and explore the improvement of knowledge about the objective reality. Each method has a peculiar approach to the object of study, which reveals different categories. This paper tries to describe a new perspective to classify these methods in accordance with the function of the research process. $A$ systematic bibliographic revision has been made to facilitate the classification of research methods used to meet the final research goals, which becomes an innovative and important outcome for research methodology. A distinctive proposal among methods that guarantee the search of information focusing on knowledge construction turns to be vital for the researcher who may use information as a tool to get oriented at the time of choosing the most pertinent method to reach project goals.
\end{abstract}

Key words. Research methods, scientific knowledge, theoretical methods, rational methods, empirical methods, empirical knowledge, rational knowledge.

\section{Méthodes scientifiques d'investigation et de construction de la connaissance}

Resumé. Les méthodes de recherche décrites dans la littérature sont un outil important pour la recherche et le perfectionnement de la connaissance de notre réalité. Chaque méthode possède sa forme particulière d'analyse de l'objet d'étude ouvrant la voie à différentes classifications. Notre travail tente de créer une nouvelle perspective de classification de ces méthodes, conformément à leur fonction dans le processus de recherche. Une systématisation des référents bibliographiques a été réalisée facilitant la caractérisation des méthodes les plus fréquemment employées et leur classification selon leur but investigatif, aspect nouveau et important pour la méthodologie de la recherche. La proposition d'une distinction entre les méthodes privilégiant la recherche d'information sur celles mises au point dans la construction de connaissance résulte d'une importance capitale pour l'investigateur qui a la possibilité d'utiliser ladite information comme un outil d'orientation au moment de sélectionner la méthode la plus pertinente en fonction de l'objectif de son projet.

Mots clefs. Méthodes de recherche, connaissance scientifique, méthodes théoriques, méthodes rationnelles, méthodes empiriques, connaissance empirique, connaissance rationnelle.

\section{Métodos científicos de indagação e de construção do conhecimento}

Resumo. Os métodos de pesquisa que se descrevem na literatura são uma importante ferramenta para a busca e o aperfeiçoamento do conhecimento a respeito da realidade. Cada método tem sua forma particular de aproximação do objeto de estudo, o que dá origem a diferentes classificações. O seguinte trabalho pretende fundamentar uma nova perspectiva de classificação destes métodos, de acordo com sua função no processo de pesquisa. Realizou-se uma sistematização de referentes bibliográficos que possibilitou caracterizar os métodos mais comumente empregados e classificá-los segundo sua finalidade investigativa, aspecto que resulta inovador e importante para a metodologia de investigação. A proposta de uma distinção entre os métodos que privilegiam a busca de informação daqueles que se focam na construção de conhecimento, resulta de vital importância para o pesquisador, que tem a possibilidade de utilizar referida informação como uma ferramenta de orientação no momento de selecionar o método mais apropriado segundo o objetivo de seu projeto.

Palabras chave. Métodos de investigação, conhecimento científico, métodos teóricos, métodos racionais, métodos empíricos, conhecimento empírico, conhecimento racional. 


\section{Introducción}

드 su acepción más general, el método es cun modo de alcanzar un objetivo, es el cómo se organiza una actividad. Como medio de cognición, es la vía empleada para reflejar en el pensamiento el objeto de estudio. Según Abbagnamo (1963), citado por Lima y De Moura (2010), el término método investigativo tiene dos significados: como orientación general -por ejemplo método dialéctico- o como técnica particular de la investigación -por ejemplo método inductivodeductivo-.

El término métodos de investigación como técnica particular se refiere a las diferentes formas en que el sujeto que investiga puede interactuar con el objeto de estudio. Los métodos que se pueden emplear en el proceso investigativo son múltiples y variados, determinados en última instancia por el objeto de estudio. Cada uno de los métodos de investigación tributa a la búsqueda y el perfeccionamiento del conocimiento acerca de la realidad y a su vez tiene su forma particular de acercamiento al objeto, lo cual puede dar lugar a diferentes criterios de clasificación.

En la literatura de la metodología de investigación científica consultada (Ortiz, 2015; Pérez, García, Nocedo de León y García, 2009, Valledor y Ceballos, 2006; Cerezal y
Fiallo, 2005; Labarca, 2001; Bisquera, 1989; Nocedo de León, 1984), la clasificación de los métodos comúnmente encontrada es aquella que los ubica como empíricos o teóricos; sin embargo, ninguno sugiere la idea de establecer un criterio taxonómico de acuerdo con dos funciones cardinales que se revelan en el proceso de investigación: la indagación o búsqueda de información y la de construcción del conocimiento para ajustar o enriquecer la estructura teórica. Por ello, se ha establecido como objetivo fundamentar una nueva perspectiva de clasificación de estos métodos de acuerdo con su función en el proceso de investigación.

Para una mejor comprensión, la estructura lógica del trabajo comienza con la presentación del método dialéctico como enfoque general, para luego esclarecer los conceptos de conocimiento empírico, racional y teórico que develarán las contradicciones existentes en la clasificación de los métodos de investigación, para posteriormente proponer un criterio taxonómico atendiendo a sus funciones en la lógica interna del proceso de investigación. Por último, como son evidentes las funciones de indagación de los métodos empíricos, se centra la atención en esclarecer la dualidad de funciones de los métodos racionales. 


\section{El método dialéctico de la investigación}

- ste constituye una metodología general - para desarrollar las investigaciones que se basan en la vía dialéctica del conocimiento de la verdad: movimiento de lo concreto sensible a lo abstracto, de este a lo concreto pensado y de este a la práctica. El punto de partida es lo concreto sensible, dado por el reflejo del mundo circundante a través de sensaciones, percepcionesy representaciones. La abstracción permite rebasar este nivel empírico mediante la separación mental de alguna o varias propiedades del objeto y sus relaciones para descubrir lo esencial oculto e inasequible al conocimiento empírico y así ascender al nivel de lo abstracto racional en que aparecen conceptos y categorías que son un reflejo de la realidad concreta en el pensamiento, por ejemplo, infinito, conciencia, materia. Pero esto no es suficiente en cuanto el papel de la ciencia es, a través de la investigación, representarse las múltiples relaciones y determinaciones de la realidad. Mediante un proceso de integración racional ocurre la conexión de las abstracciones en el pensamiento con fines de generalización y de esta manera se alcanza lo concreto pensado, que refleja el enlace y las múltiples dependencias entre los hechos, los procesos y los fenómenos y las contradicciones que condicionan su funcionamiento y desarrollo. Lo concreto pensado se manifiesta en principios, leyes y teorías que posteriormente deben someterse a constatación empírica para corroborar su objetividad en la práctica.

\section{Conocimiento empírico, racional y teórico _.}

- mpírico significa referente a la experiencia. Se refiere al uso de los sentidos, tanto en la observación de los objetos y fenómenos como en la experimentación o manipulación física de ellos. Los sentidos y el aspecto físico de las cosas están en el primer plano de la atención.

Según Cerezal y Fiallo (2005), el conocimiento empírico es aquel tomado de la práctica, analizado y sistematizado por vía experimental mediante la observación reiterada y la experimentación. Constituye la primera etapa del conocimiento, donde el hombre obtiene el reflejo del mundo circundante a través de sensaciones, percepciones y representaciones.

Según estos autores, el conocimiento teórico constituye el segundo nivel, donde, mediante los procesos lógicos del pensamiento, el hombre analiza, sintetiza, generaliza y extrae conclusiones sobre la esencia y los vínculos internos de los procesos, hechos y fenómenos, para explicarlos y descubrir las leyes que los rigen y poder agruparlos en un sistema único que son las teorías.

A juicio de los autores de este trabajo, este segundo nivel es más bien racional que teórico, pues, en realidad, ambos niveles conforman una unidad en el conocimiento teórico, como ha quedado evidenciado a través del movimiento de lo concreto sensible a lo abstracto y de este a lo concreto pensado. El primer nivel -conocimiento empírico- se corresponde con lo abstracto concreto y el segundo con lo abstracto y lo concreto pensado. Los dos niveles están estrechamente relacionados y conforman un todo: conocimiento objetivo de la realidad, que es sinónimo de conocimiento teórico. 
Por tanto, si quisiéramos distinguir la segunda y tercera etapa de la primera, sería más apropiado hablar en la primera de conocimiento empírico y en la segunda y tercera de conocimiento racional. Visto así, se puede plantear que el proceso de construcción de conocimientos ocurre a través de tres etapas -concreto sensible, abstracto y concreto pensado-, donde se distinguen dos niveles de conocimiento -el empírico y el racional(Figura 1).

Figura 1. El conocimientos científico.

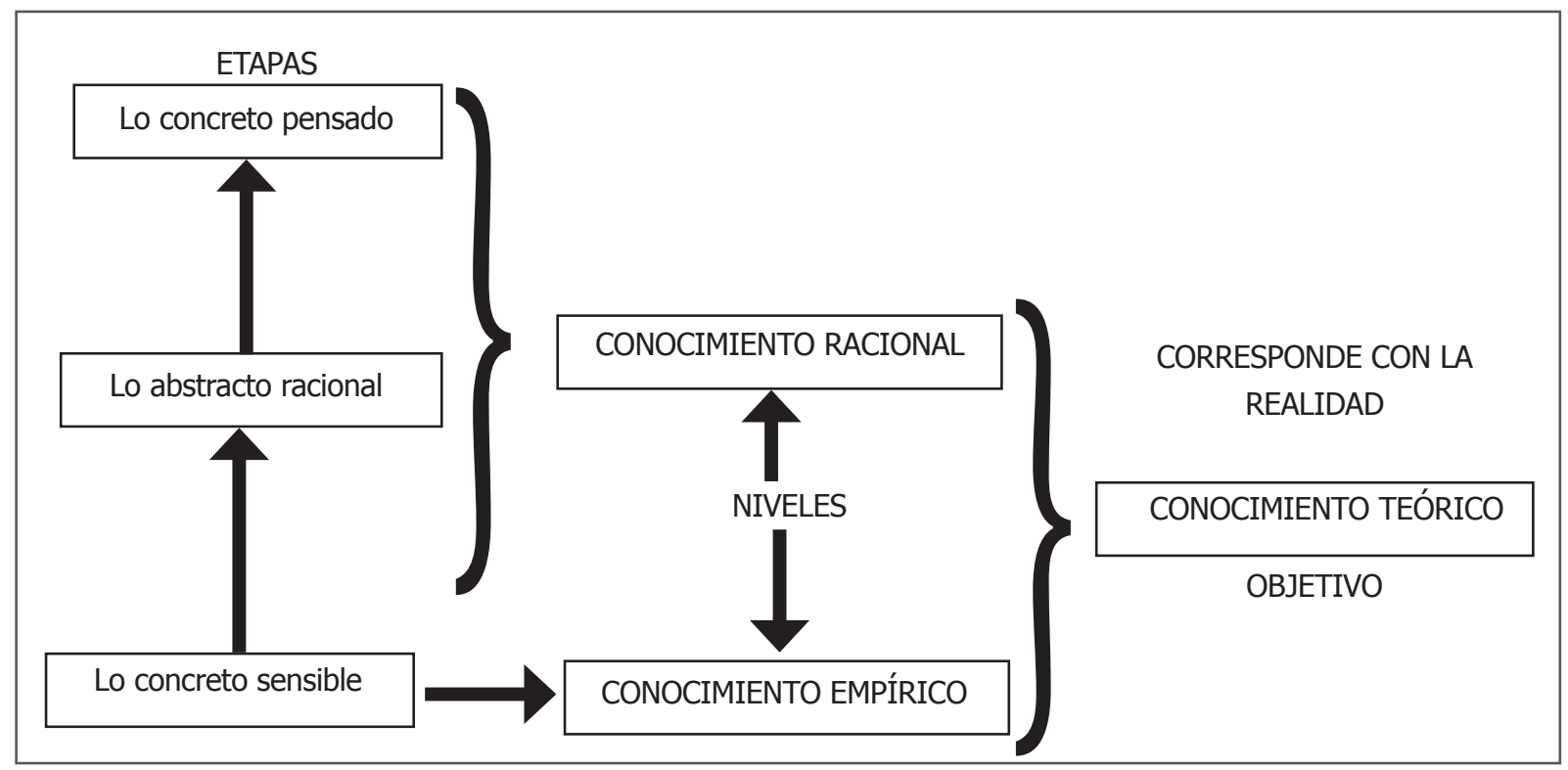

Fuente. Elaboración propia de los autores.

La importancia de lo empírico en el método científico se evidencia principalmente porque pone en contacto con los objetos y fenómenos reales, proporciona pistas para formular hipótesis y datos para la construcción de conocimientos y conduce a la verificación de las hipótesis previamente formuladas. El dato empírico proporciona las pistas para llegar al conocimiento racional, pero no debe confundirse ese dato singular con la unidad producida en el concepto, ni mucho menos con las derivaciones producidas en el raciocinio. El significado de esos datos singulares y sus relaciones se obtienen por medio del entendimiento o razón. Por ello, además del nivel sensible, el método científico para la construcción del conocimiento requiere un aspecto o nivel racional. El nivel racional se refiere al uso de la razón para la búsqueda de información teórica, la elaboración de hipótesis, conceptos, leyes y teorías, la expresión abstracta de los resultados empíricos y para la inferencia de conclusiones; por ejemplo, la hipótesis es una relación universal y su formulación no podría realizarse con base exclusiva en el conocimiento empírico, sino que requiere una actividad superior: el raciocinio.

Lo empírico no necesariamente se opone a lo racional, pero sí son dos niveles diferentes en la construcción del conocimiento que a su vez forman una unidad dialéctica. Por tanto, se requiere una postura equilibrada que reconozca ambos niveles de conocimiento y deslinde y acepte el valor propio de cada uno de ellos, sin privilegiar uno de estos en detrimento del otro, porque conforman una unidad inseparable en la elaboración de teorías. 


\section{Clasificación de los métodos de investigación científica}

ay coincidencia entre varios autores (Álvarez de Zayas, 1993; Lanuez, Martínez y Pérez (s. f.); Notario de la Torre, 1999; Gallo y González, 2002; Rosales, 2002; Cerezal y Fiallo, 2005; Ramírez, 2008; Valledor y Ceballos, 2005; Pérez et al., 2009), en

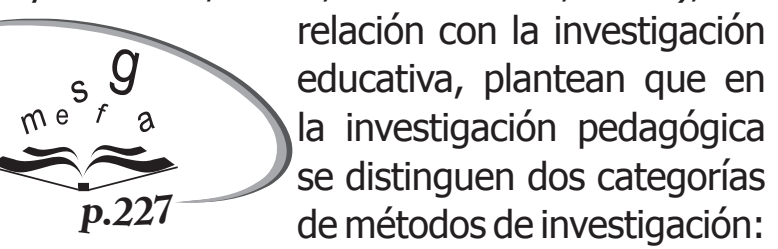
los empíricos y los teóricos, y tanto uno como otro se apoyan en los métodos matemáticoestadísticos.

Siguiendo la idea anterior del conocimiento empírico y del conocimiento racional, consideramos que sería más apropiado hablar de métodos empíricos y métodos racionales, pues, de acuerdo con este razonamiento, los métodos empíricos también se emplean para la elaboración de las teorías y desde este punto de vista también serían métodos teóricos.

Los autores consultados coinciden en que los métodos empíricos se utilizan para descubrir y acumular un conjunto de hechos y datos como base para verificar la hipótesis, dar respuesta a las preguntas científicas de la investigación, obtener argumentos para defender una idea o seguir una guía temática, pero que no son suficientes para profundizar en las relaciones esenciales que se dan en los procesos pedagógicos.

Son los métodos racionales los que posibilitan sistematizar y analizar los resultados obtenidos con métodos empíricos, descubrir lo común y arribar a conclusiones en relación con la solución del problema científico, pues los empíricos proporcionan las pistas para la elaboración de las teorías mediante los racionales, y además, una vez formuladas las teorías, posibilitan su confirmación en la realidad.

\section{Finalidades de los métodos investigativos que atienden a la lógica interna de la investigación científica}

D érez (2014a), establece cuatro momentos diferentes para la lógica interna de la investigación: i) precisión del problema, ii) red de indagaciones, iii) ajuste o conformación del sistema teórico, conceptual o metodológico; y iv) constatación práctica. En cada uno de esos momentos, el investigador emplea variados métodos que le permiten explorar lo multifacético del objeto. En este proceso, se combinan los métodos empíricos y los racionales, aunque en determinados momentos predominan unos o los otros y pueden tener dos finalidades diferentes: la búsqueda y el procesamiento de la información necesaria como parte de la red de indagaciones o la elaboración de nuevos conocimientos para el momento del ajuste o conformación del sistema teórico, conceptual o metodológico. 
Los empíricos se emplean fundamentalmente en la precisión del problema, en un primer momento de la investigación para la acumulación de datos, hechos, testimonios, etc., y finalmente en la constatación práctica. Entre ellos, se destacan la observación, el experimento, la encuesta, la entrevista y la prueba pedagógica. Los métodos empíricos, en general, responden a la finalidad de la búsqueda de información y por tanto se asocian al momento de la red de indagaciones.

Por su parte, los métodos racionales están presentes desde que comienza la preocupación por un problema de la práctica social, se estudian sus manifestaciones, las posibles causas que pueden generarlo, se indaga sobre estudios realizados en la temática y, como resultado de ello, se precisa el problema que pasa por diseño de la investigación, análisis de los datos empíricos, realización de inferencias, reconstrucción del marco conceptual o metodológico, hasta el momento en que se elaboran las conclusiones y recomendaciones.

Partiendo de esta premisa, los métodos racionales tienen como finalidades investigativas esenciales la búsqueda, el uso y la transformación de la información empírica y teórica cuando forman parte de la red de indagaciones y la construcción de nuevos conocimientos cuando intervienen en el momento del ajuste o conformación del sistema teórico, conceptual o metodológico. Aunque en el cumplimiento de una de estas finalidades se pueden combinar diferentes métodos racionales, algunos cumplen esencial-mente la primera finalidad y otros la segunda.

Un mismo método racional en un caso puede ser empleado para la búsqueda y procesamiento de información -referentes teóricos, metodológicos y prácticos- y en otro para la elaboración de conocimientos; pero el empleo más común en las investigaciones, con una finalidad o la otra, podría ser la base para clasificarlo esencialmente en uno de los dos grupos -métodos para la búsqueda de información o métodos para la construcción de conocimientos-, siempre y cuando se tenga en cuenta que esta clasificación no es rígida, sino que tiene un carácter flexible. Los primeros responderían esencialmente al momento de la red de indagaciones y los últimos al momento del ajuste o conformación del sistema teórico, conceptual o metodológico.

Entre los métodos racionales más reconocidos y estudiados, se encuentran el analítico-sintético, el inductivo-deductivo, el método de analogías, el hipotético-deductivo, el histórico lógico, el sistémicoestructural-funcional, la sistematización, el genético y la modelación. En lo que sigue se trata de fundamentar la clasificación de cada uno de ellos en el grupo de los que tributan fundamentalmente a la búsqueda de información o de los que lo hacen a la construcción de conocimientos. 


\section{Los métodos racionales y su clasificación atendiendo a la finalidad a la que se orientan en los diferentes momentos de la lógica investigativa}

\subsection{Método analítico-sintético}

Este método se refiere a dos procesos intelectuales inversos que operan en unidad: el análisis y la síntesis. El análisis es un procedimiento lógico que posibilita descomponer

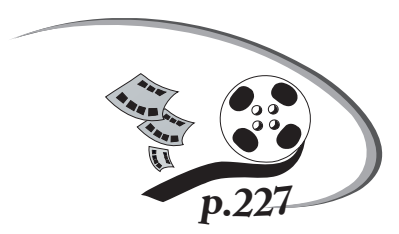

mentalmente un todo en sus partes y cualidades, en sus múltiples relaciones, propiedades y componentes. Permite estudiar el comportamiento de cada parte. La síntesis es la operación inversa, que establece mentalmente la unión o combinación de las partes previamente analizadas y posibilita descubrir relaciones y características generales entre los elementos de la realidad. Funciona sobre la base de la generalización de algunas características definidas a partir del análisis. Debe contener solo aquello estrictamente necesario para comprender lo que se sintetiza.

El análisis y la síntesis funcionan como una unidad dialéctica y de ahí que al método se le denomine analítico-sintético. El análisis se produce mediante la síntesis de las propiedades y características de cada parte del todo, mientras que la síntesis se realiza sobre la base de los resultados del análisis. En la investigación, puede predominar uno u otro procedimiento en una determinada etapa.

El análisis y la síntesis no son el resultado del pensamiento puro, sino que tienen una base objetiva en la realidad. Como el propio mundo es a la vez único y múltiple, los objetos, fenómenos y procesos de la realidad también lo son. Ello nos lleva a que cada objeto, fenómeno o proceso está constituido por partes que tienen sus identidades y deferencias entre sí, pero que a su vez establecen interacciones que dan las características del todo. El conocimiento de la realidad objetiva entonces requiere la descomposición mental -análisis- del objeto, proceso o fenómeno en sus múltiples partes (lo múltiple en lo único) que se realiza mediante la síntesis, y la integración de las partes en los objetos y fenómenos estudia lo único en lo múltiple a través de la síntesis de lo analizado.

Tratando de clasificar este método racional según la finalidad investigativa, a partir del uso más común que de él hacen diferentes autores en sus investigaciones, se encuentra que lo más frecuente es que se le emplee para la búsqueda y el procesamiento de la información, como se puede apreciar en los ejemplos que se citan a continuación:

Suárez (2014), expresa que empleó este método para resumir la búsqueda bibliográfica. Herrera, Herrera y Pérez (2012), plantean que utilizaron el método para facilitar el análisis y la clasificación de las fuentes de información recopiladas en busca de la esencia de las ideas. Martínez (2016), precisa que el método se utilizó para analizar la documentación referente al tema de investigación, lo cual permitió la extracción de los elementos más importantes que se relacionan con el objeto de estudio. 
Sin embargo, en algunos casos, se encuentra que el método, sobre todo en su momento de síntesis, es utilizado para construir conocimientos, como se puede inferir a partir del siguiente ejemplo:

Véliz y Jorna (2014), expresan que el método analítico-sintético fue empleado para descomponer el todo en las partes, conocer las raíces $y$, partiendo de este análisis, realizar la síntesis para reconstruir y explicar. Aquí la reconstrucción y explicación implican elaboración de conocimientos, lo cual es un llamado a que, aunque lo más común en su empleo es para la búsqueda de información, en ocasiones se le utiliza para la elaboración de conocimientos.

A nuestro juicio, el método analíticosintético tiene gran utilidad para la búsqueda y el procesamiento de la información empírica, teórica y metodológica. El análisis de la información posibilita descomponerla en busca de lo que es esencial en relación con el objeto de estudio, mientras que la síntesis puede llevar a generalizaciones que van contribuyendo paso a paso a la solución del problema científico como parte de la red de indagaciones necesarias; pero, como método singular, generalmente, no se emplea para la construcción de conocimientos. Aunque cuando forma parte de un método más complejo, como el sistémico estructuralfuncional, las generalizaciones a que se arriban mediante la síntesis pueden constituir regularidades, principios o leyes que conforman una teoría, su finalidad predominante es la búsqueda de información.

\subsection{Método inductivo- deductivo}

El método inductivo-deductivo está conformado por dos procedimientos inversos: inducción y deducción. La inducción es una forma de razonamiento en la que se pasa del conocimiento de casos particulares a un conocimiento más general, que refleja lo que hay de común en los fenómenos individuales. Su base es la repetición de hechos y fenómenos de la realidad, encontrando los rasgos comunes en un grupo definido, para llegar a conclusiones de los aspectos que lo caracterizan. Las generalizaciones a que se arriban tienen una base empírica.

Francis Bacon (1561-1626), citado por Dávila (2006), fue el primero que propuso la inducción como un nuevo método para adquirir conocimientos. Afirmaba que para obtener conocimiento es imprescindible observar la naturaleza, reunir datos particulares y hacer generalizaciones a partir de ellos. Según Bacon, las observaciones se hacían sobre fenómenos particulares de una clase y luego a partir de ellos se hacían inferencias de la clase entera. Este procedimiento es lo que hoy se denomina razonamiento inductivo, que habría de convertirse en el principio fundamental de todas las ciencias. Sus pasos son estos: i) observación; ii) formulación de hipótesis; iii) verificación; iv) tesis; v) ley y vi) teoría.

Estemétodo ha sido muy efectivo a lo largo de la historia para avanzar en el conocimiento científico en las áreas de las ciencias naturales y exactas. Es el método base del paradigma positivista de investigación, pues, al partir de evidencias empíricas, alegan sus defensores, está despojado por completo del subjetivismo. Este procedimiento inductivo ha sido potenciado por los positivistas como el único válido para generar conocimientos y 
en la actualidad también se utiliza con mucha frecuencia por las ciencias sociales como vía esencial de construir conocimientos desde el terreno investigativo (Charmaz, 2006). Pero ello no debe llevar a confusión, porque las ciencias sociales involucran otros fenómenos que difieren de los que son objeto de estudio de las ciencias naturales y por tanto su aplicación mecánica puede conducir a conclusiones que se alejen de la realidad estudiada.

Otro procedimiento utilizado para la obtención de conocimientos es el razonamiento deductivo. Tuvo su origen entre los filósofos griegos. Aristóteles y sus discípulos lo implantaron como un proceso del pensamiento en el que, de afirmaciones generales, se llegaba a afirmaciones particulares que aplicaban las reglas de la lógica. Mediante este procedimiento, se organizan hechos conocidos y se extraen conclusiones mediante una serie de enunciados, conocidos como silogismos, que comprenden: la premisa mayor, la premisa menor y la conclusión (Dávila, 2006); por ejemplo, todos los seres vivos son mortales (premisa mayor), los corales son seres vivos (premisa menor), por tanto, los corales mueren (conclusión).

Mediante la deducción se pasa de un conocimiento general a otro de menor nivel de generalidad. Las generalizaciones son puntos de partida para realizar inferencias mentales y arribar a nuevas conclusiones lógicas para casos particulares. Consiste en inferir soluciones o características concretas a partir de generalizaciones, principios, leyes o definiciones universales. Se trata de encontrar principios desconocidos, a partir de los conocidos o descubrir consecuencias desconocidas, de principios conocidos; por ejemplo, obtener conclusiones prácticas referentes al comportamiento de alguna sustancia, en función de un principio o ley general que allí se aplica. La matemática es la ciencia deductiva por excelencia; parte de axiomas y definiciones para estudiar casos particulares.

Así, de lo más general, se realizan deducciones lógicas que originan nuevas regularidades, principios y leyes de menor grado de generalidad que las de partida. De esta forma, se reestructura o reajusta el sistema teórico, conceptual o metodológico de la propuesta de solución al problema científico. Así, el conocimiento se integra en un sistema con una estructura jerarquizada de regularidades, principios y leyes, pues en la cima de esta estructura se situarían las regularidades, los principios y las leyes de mayor grado de abstracción, generalidad y fuerza lógica, a partir de los cuales se deducen los que contienen en menor grado estos atributos.

La inducción y la deducción se complementan mutuamente: mediante la inducción se establecen generalizaciones a partir de lo común en varios casos, luego a partir de esa generalización se deducen varias conclusiones lógicas, que mediante la inducción se traducen en generalizaciones enriquecidas, por lo que forman una unidad dialéctica. De esta manera, el empleo del método inductivodeductivo tiene muchas potencialidades como método de construcción de conocimientos en un primer nivel, relacionado con regularidades externas del objeto de investigación. 


\subsection{Método hipotético- deductivo}

En este método, las hipótesis son puntos de partida para nuevas deducciones. Se parte de una hipótesis inferida de principios 0 leyes o sugerida por los datos empíricos, y aplicando las reglas de la deducción, se arriba a predicciones que se someten a verificación empírica, y si hay correspondencia con los hechos, se comprueba la veracidad o no de

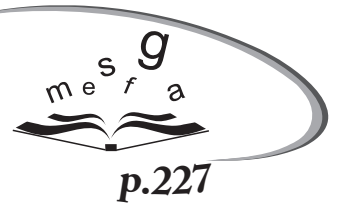

la hipótesis de partida. Incluso, cuando de la hipótesis se arriba a predicciones empíricas contradictorias, las conclusiones que se derivan son muy importantes, pues ello demuestra la inconsistencia lógica de la hipótesis de partida y se hace necesario reformularla.

Este método es de uso muy común en medicina, donde se identifica como diagnóstico clínico. León-Barúa (1999) resume los pasos fundamentales de este diagnóstico: al consultar a un médico sobre lo que siente, el paciente le refiere sus síntomas -historia clínica- y, además, el médico lo examina para indagar sobre los cambios corporales producidos por la enfermedad -examen físico-. Sobre la base de los datos obtenidos mediante la historia clínica y el examen físico, el médico, considerando conocimientos previos acumulados en las ciencias médicas, se plantea una o varias hipótesis -posibilidades diagnósticas- que expliquen la génesis del problema que aqueja al paciente. Esa hipótesis o esas hipótesis son luego puestas a prueba con ayuda de exámenes auxiliares. El resultado de los exámenes auxiliares efectuados verifica o descarta las posibilidades diagnósticas planteadas.
Esta forma corriente de proceder del médico ilustra con claridad la metodología general con que opera el método hipotéticodeductivo. La esencia del método consiste en hacer uso de la verdad o falsedad del enunciado básico -a partir de su constatación empírica-, para inferir la verdad o la falsedad de la hipótesis que ponemos a prueba. Requiere el empleo de los más exigentes contraejemplos y determinar si se cumplen o no. Refutar estos contraejemplos significa demostrar la veracidad de la hipótesis (Behar, 2008).

Este método posibilita la reestructuración constante del sistema teórico, conceptual o metodológico de la investigación y, por tanto, se puede clasificar esencialmente como método para la construcción de conocimientos.

\subsection{Método histórico-lógico}

Lo histórico se refiere al estudio del objeto en su trayectoria real a través de su historia, con sus condicionamientos sociales, económicos y políticos en los diferentes periodos. Lo lógico interpreta lo histórico e infiere conclusiones. La combinación de lo histórico con lo lógico no es una repetición de la historia en todos sus detalles, sino que reproduce solo su esencia.

Lo histórico y lo lógico están estrechamente vinculados. Lo lógico para descubrir la esencia del objeto requiere los datos que le proporciona lo histórico. De otra manera, se trataría de un simple razonamiento especulativo. Sin embargo, lo lógico debe reproducir la esencia y no limitarse a describir los hechos y datos históricos. Estas ideas se resumen en que lo lógico es lo histórico liberado de la forma histórica. 
Se debe reconocer esta unidad dialéctica histórico-lógico y rechazar tanto el razonamiento lógico especulativo, divorciado de los hechos científicos, como el empirismo que se limita a la simple descripción de los hechos sin explicarlos a partir de la lógica de su desarrollo.

La consulta de informes de investigación de diferentes autores, como los que se refieren a continuación, posibilita arribar a conclusiones acerca de la finalidad con que más comúnmente se emplea este método racional en las investigaciones:

De la Uz Herrera, Lemus, Valdés y Padrón (2010), plantean que emplearon este método para hacer una síntesis teórica del proceso de formación de la competencia comunicativa en el individuo en su desarrollo y de las regularidades y componentes de este proceso.

Jivkova (2011), expresa que empleó el método histórico-lógico para realizar una caracterización del comportamiento en los últimos años de la situación económicofinanciera de la radiotelevisión pública en España, en busca, al mismo tiempo, de ejemplos de gestión de países como Francia, el Reino Unido y Alemania.

González (2008) declara que aplicó este método para establecer el estado del tema de investigación. Mediante él se estudió la existencia de sistemas informáticos similares o relacionados con el tema en cuestión y su comportamiento en el decurso del tiempo, lo cual permitió profundizar en las particularidades de estos y sacar experiencias propias para la futura aplicación.

Castro (2010) afirma que empleó este método para analizar los antecedentes, las causas y las condiciones en que se ha desarrollado el análisis de la contabilidad y los costos medioambientales o ecológicos.

Los ejemplos presentados son una muestra de que lo más común en el uso del método está en la búsqueda de información para tratar de forma lógica los antecedentes relacionados con el objeto de estudio. Este hecho no niega que, en algunos casos, la aplicación de la lógica podría llevar a la revelación de nuevos conocimientos.

El análisis de la práctica investigativa posibilita afirmar que este método se emplea comúnmente cuando se buscan los antecedentes del problema científico y durante la elaboración de los fundamentos teóricos y metodológicos de la propuesta de solución al problema. En ambos casos, su finalidad es la búsqueda de información como parte del momento de la red de indagaciones.

\subsection{Método genético}

Es una forma de concreción del método histórico-lógico, pues se ocupa de estudiar el objeto en su evolución y los factores que lo condicionan. Implica la determinación de una célula básica del objeto de estudio, donde están presentes todos los componentes del objeto en interacción; por ejemplo, para el estudio de un organismo vivo, sería la célula.

A fin de llevar a cabo la investigación, se pude realizar un estudio longitudinal o transversal. En el longitudinal se investiga la célula básica en una muestra a través de su evolución en un periodo determinado y tiene como desventaja que requiere mucho tiempo para obtener los resultados. En el estudio transversal, se utiliza una muestra de diferentes sujetos que se encuentran en 
distintos niveles o estadios de desarrollo para acortar el tiempo de obtención de los resultados, pero tiene como desventaja que no se puede seguir la evolución de la célula básica; por ejemplo: velocidad de cálculo matemático oral en diferentes edades, desde los cinco hasta los quince años. Si se hace un estudio longitudinal la muestra que se selecciona se sigue periódicamente desde los cinco hasta los quince años. Si se realiza un estudio transversal, la muestra se toma estratificada por edades para tener una idea rápida de cómo evoluciona la célula básica. Este método es esencialmente empleado para la búsqueda de información acerca del objeto de estudio $y$, en este empeño, pueden combinarse estudios longitudinales $y$ transversales.

\subsection{Método de analogías}

Consiste en inferir relaciones o consecuencias semejantes en fenómenos parecidos; por ejemplo, conozco un fenómeno a y su consecuencia $b$, por otro lado, estudio un fenómeno $x$ cuya semejanza con a es evidente. A partir de esto, propongo una consecuencia y semejante o análoga a la consecuencia $b$.

El razonamiento por analogía puede producir conclusiones válidas. Sin embargo, tiene sus limitaciones:

- Los comportamientos análogos se presentan cuando no intervienen determinados factores -variables extrañas- que pueden encauzar al fenómeno en otro sentido y la eliminación de esos factores no siempre es fácil ni posible.
- La semejanza entre los dos fenómenos puede ser aparente y no real. Para que sea real, la analogía entre los dos fenómenos debe abarcar no solo aspectos superficiales, sino de fondo. Un análisis minucioso podría verificar si el parecido entre los dos fenómenos es real.

Aun en caso de analogías reales entre los fenómenos y la no existencia de variables extrañas, las inferencias a que se arribe sería necesario someterlas a verificación empírica (Pérez, 2014b).

El empleo del método de analogías ha sido utilizado por las ciencias para construir nuevos conocimientos. Así, James Clerk Maxwell logró, a partir del empleo de analogías mecánicas, entender la naturaleza de los campos electromagnéticos y concebir una teoría unificada del magnetismo, la electricidad y la luz (Cachón, 2013). Este método es muy útil en el campo de la ingeniería geológica para estimar los incrementos y decrecimientos del grado sísmico regional con respecto al suelo promedio (Ordaz-Hernández, Chuy-Rodríguez y García-Gutiérrez, 2011).

En el área de las investigaciones sociales, por analogía, se pueden inferir consecuencias semejantes en épocas históricas parecidas y también se puede presumir un comportamiento determinado en una persona por la semejanza que presenta con otra ya conocida.

Con independencia de las limitaciones que en la construcción de conocimientos este método pueda tener, su finalidad predominante como método racional es esta, aunque en determinados momentos también pueda ser útil en la búsqueda de información. 


\subsection{Método de modelación}

En este método, se crean modelos para investigar la realidad. El modelo debe tener cierta analogía estructural y funcional con el objeto de investigación. Valle (2007), señala que es una de las vías más importantes en la adquisición de nuevos conocimientos. Según este autor, el proceso de modelación comienza con la obtención de una abstracción de la realidad -modelo- que se materializa, se trabaja con esta materialización y se obtiene un nuevo conocimiento que se aplica a la explicación de la realidad de la cual se partió. A partir de tal lógica, propone un sistema de acciones para emplear este método: análisis del problema planteado, creación -eleccióndel modelo, materialización del modelo, la investigación o trabajo con la materialización del modelo, obtención del nuevo resultado y extrapolación del nuevo resultado al problema inicial.

Se comienza por formular el objetivo y, a partir de este, se separa lo esencial de lo no esencial del objeto. Ello permite establecer una idea clara de la esencia del objeto y esa abstracción de la realidad constituye el modelo. Una vez obtenido el modelo se comienza a buscar una posible materialización, de forma tal que esta sea más simple que el objeto inicial y que se simplifique el trabajo del investigador.

En la siguiente etapa, la materialización del modelo deviene objeto de investigación. En este caso, todas las acciones producidas sobre ella están encaminadas a la obtención de nuevos conocimientos y el establecimiento de las leyes de su desarrollo, de sus propiedades y relaciones. Como elemento final de este trabajo, se obtiene un nuevo resultado que debe ser confrontado con la realidad, con el problema inicial.
Al determinar lo esencial del objeto, ocurre el proceso de abstracción, a través de la cual se pasa de lo concreto sensible (objeto de estudio) a lo abstracto (el modelo y su materialización). La investigación con la materialización del modelo se identifica con el proceso de integración para transitar hacia lo concreto pensado, en el que se elaboran los nuevos conocimientos mediante la ejecución de acciones mentales sobre la materialización lograda. El elemento final es el que cierra el ciclo del conocimiento: práctica-teoríapráctica y que se emplea para constatar el grado de objetividad de los conocimientos elaborados.

Como se aprecia, este método es fiel al método dialéctico de obtención del conocimiento: de lo concreto sensible a lo abstracto, de este a lo concreto pensado y por último la confrontación del conocimiento elaborado con la realidad.

Como plantean Reyes y Bringas (2006), la modelación es un proceso complejo que demanda una forma superior de construcción teórica, pues requiere una elevada capacidad de abstracción. No cabe duda de que la finalidad de este método es la construcción de conocimientos como parte del ajuste o conformación del sistema teórico, conceptual o metodológico del proceso de investigación.

\subsection{Método sistémico- estructural-funcional}

Este método proporciona una orientación general para la investigación del objeto de estudio como una realidad que es a la vez única y compuesta. Tuvo su génesis epistemológica desde la Antigüedad. Así, para Aristóteles, el todo era más que la suma de las partes, pues las interacciones de las partes 
en el todo producían cualidades que no eran el resultado de la sumatoria mecánica de las cualidades de cada parte.

Este método de investigación ha tenido una gran influencia del estructuralismo como corriente filosófica que tuvo su máximo auge en el siglo XX. Según Piaget (1968), en el estructuralismo se hace hincapié en la interrelación e interdependencia entre las partes del todo que posibilitan la autorregulación y que asegura tanto la estabilidad del sistema en cuestión como su transformación. La estructura es el modo en que dichas partes se conectan entre sí en el todo y forma un esqueleto en el que se distingue lo secundario de los elementos indispensables. El estructuralismo insiste en determinar cuáles son los elementos esenciales, determinantes, profundos, para distinguirlos de aquellos secundarios o superficiales de un fenómeno. En esta concepción, el todo es el sistema o estructura.

El término estructural-funcional tuvo su origen en la demostración, con argumentación suficiente en los estudios biológicos, de la interdependencia imprescindible entre la estructura de cualquier órgano y las funciones que desempeña en un organismo vivo. Este análisis se trasladó al enfoque de determinadas ciencias sociales, en las que el método estructural-funcionalista se convirtió en una herramienta heurística de extraordinario valor.

En este sentido, no se debe perder de vista que un enfoque dialéctico materialista es opuesto a la estabilidad y permanencia del sistema que se resalta en un enfoque puramente estructural y funcional, pues a su vez cada sistema es un subsistema de otro mayor, donde existen otros componentes que ejercen su influencia sobre el subsistema objeto de estudio, en el que se evidencia lo que afirma Morin (2002) en su teoría de la complejidad:

\begin{abstract}
Todas las cosas son ayudadas y ayudantes, todas las cosas son mediatas e inmediatas, y todas están ligadas entre sí por un lazo que conecta unas a otras, aun las más alejadas. En esas condiciones considero imposible conocer las partes si no conozco el todo, pero considero imposible conocer el todo si no conozco las partes (p. 422).
\end{abstract}

En la investigación científica, el método sistémico estructural funcional está dirigido a modelar el objeto como sistema, para lo que se deben determinar componentes, estructura, principio de jerarquía y las relaciones funcionales. Como opera sobre la base de la modelación, en su aplicación, son válidos los análisis realizados para la elaboración de modelos de investigación y, por tanto, su finalidad fundamental es la construcción de conocimientos.

\subsection{Método de sistematización}

El método de sistematización ha estado ligado al desarrollo del método científico. Sus usos más comunes son en la sistematización de información o datos y en la sistematización de experiencias. El primero se refiere al ordenamiento y la clasificación de datos e información y el segundo a procesos que se desarrollan en un periodo determinado, en un contexto económico-social y dentro de una institución dada.

Según la Guía metodológica para la sistematización participativa de experiencias en agricultura sostenible (Ardón, 2000), la inquietud por sistematizar experiencias surgió 
en la década de 1980 en los profesionales que trabajaban con grupos sociales. Sin embargo, la historia de las ciencias muestra que este método ha sido generador de conocimientos científicos desde mucho antes. Así, por ejemplo, la ley periódica formulada por Mendeleiev en el siglo XIX, surgió de una sistematización de la experiencia como profesor de Química de su descubridor.

Un estudio en la literatura de lo que expresan diferentes autores acerca de este método revela la existencia de variadas definiciones. Según Zúñiga (1992), la sistematización deriva de sistema y este último término implica orden, unidad, coherencia, articulación, integración de partes, conjuntos de relaciones, de interacciones. Estudiar la realidad como sistema es un modo de captarla simultáneamente unificada y compuesta.

Esta concepción de sistema subyace en la mayoría de las definiciones de sistematización de experiencias consultadas. Así, por ejemplo, Jara (1998) plantea que la sistematización es aquella interpretación crítica de una o varias experiencias que, a partir de su ordenamiento y reconstrucción, descubre o explicita la lógica del proceso vivido, los factores que han intervenido en dicho proceso, cómo se han relacionado entre sí, y por qué lo han hecho de ese modo (p. 10).

Visto así, la experiencia, al ser sistematizada, permite obtener conocimiento. Esta conceptualización presupone que la experiencia es el punto de partida, pero no aclara que la interpretación crítica de ella requiere, primero, un posicionamiento teórico del que investiga para interrogarla científicamente, que sí se hace evidente en la siguiente definición brindada por Quirós y Rodríguez (2004):
Proceso de reflexión e interpretación crítica de una intervención profesional o de un aspecto de ella, que parte de la explicitación del marco epistemológico, teórico y valorativo desde el cual se intervino y desde el cual se realizará la reflexión sean o no semejantes (p.11).

Del análisis de esta definición queda clara la posición de las autoras en relación con la metodología de la sistematización, que en este caso no partiría de la experiencia, sino de la interpretación teórica del marco desde el cual se proyecta y se realiza la experiencia. Compartimos estas ideas, pues todo sujeto que decide estudiar la realidad lo hace desde conocimientos, creencias y representaciones previas. Dejar todo esto de lado para proclamar objetividad del conocimiento no será posible. Siempre realizará sus observaciones desde alguna posición teórica y sus elecciones serán el resultado de cierta identificación o preferencia que le hace considerar una teoría más apropiada que otra. Aun cuando se declare que la experiencia es el punto de partida para la sistematización, el acercamiento a esa realidad se hace desde un posicionamiento teórico que es lo que permite al sujeto establecer nuevas relaciones y construir nuevas denominaciones mediante el uso de la razón.

Esta relación entre teoría y experiencia es dialéctica:

Mientras que esta última origina conocimientos que, sistematizados, fecundan la teoría, es esta quien propicia la interpretación, explicación y proyección de la práctica, conformando de esta forma un binomio constantemente atravesado por la crítica y la reflexión. (Rodríguez del Castillo, s. f.). 
Si bien no es la realidad por sí sola la que origina el conocimiento, sino que son los sujetos quienes delimitan esa realidad, la interpretan y le dan significado a partir de un marco racional, ello no presupone un pensamiento estático. Es necesario considerar el carácter dialéctico que tiene esa relación entre la realidad y el conocimiento preexistente en el sujeto que la investiga. El conocimiento es desde donde el sujeto interpreta y reflexiona críticamente la realidad, mientras que esta última, por una parte, es la que ha formado al sujeto y por tanto condiciona su modo de interpretar y reflexionar; y por otra, limita y restringe el conocimiento, evitando interpretaciones muy alejadas de ella (Bernaldo de Quirós y Rodríguez, 2004).

Por lo analizado hasta aquí, podemos concluir que la sistematización como método racional de investigación puede tener dos finalidades diferentes: i) la sistematización como herramienta de procesamiento de información y ii) la sistematización de experiencias; por tanto, será menester aclararlo cuando nos refiramos a este método.

Por una parte, como herramienta para procesar información, puede quedarse en elementos sistematizados o puede originar nuevos conocimientos. Por ejemplo, la sistematización de varias teorías posibilita la formulación de nuevas perspectivas. Por otra parte, como sistematización de experiencia, la práctica pedagógica de un docente puede llevar a su ejecución por parte de otros o puede conllevar generalizaciones valiosas que enriquezcan el conocimiento (Rodríguez del Castillo, s. f.).

Atendiendo a los momentos de la lógica investigativa, la sistematización en cualesquiera de sus variantes podría responder al momento de la red de indagaciones o al del reajuste o elaboración del marco conceptual, teórico o metodológico de la investigación, lo cual dependerá del objetivo con que se emplee.

El empleo de la sistematización para la construcción de conocimientos requiere (Rodríguez del Castillo, s. f.):

- Determinación del objetivo, objeto y eje de sistematización.

- Localización, ordenamiento, clasificación, análisis e interpretación de la información.

- Conclusión de las principales ideas que hayan surgido en el orden conceptual en su relación con el saber constituido, lo cual incluye conjeturas que tributen a generalizaciones de mayor alcance.

Antillón (2002), aclara acerca de estos términos y pasos. El objetivo debe permitir delimitar el objeto de sistematización dentro de una obra, teoría, experiencias, etc. El objeto se trata de un primer corte a la experiencia -tema, espacio y tiempo-. El eje de sistematización posibilita centrar el estudio en lo que se decida para así resaltar ese aspecto. En el análisis e interpretación de la información, se deben tener en cuenta la descripción de los hechos significativos para quienes vivieron el proceso -lo vivencial-, el establecimiento de las contradicciones que se fueron dando, asumir fundamentos teóricos, relacionar las partes con el todo, la toma de distancia, etc. Las conclusiones deben ser concretas y pueden referirse a las lecciones que se aprenden, lo que se aporta a un área del conocimiento, etc.

A modo de resumen, se puede expresar que los métodos empíricos, salvo contadas 
excepciones, son utilizados en la búsqueda de información; sin embargo, no siempre los racionales constituyen métodos para la construcción del conocimiento, pues algunos tienen la función esencial de la búsqueda de información. Atendiendo a la función que predomina en el proceso de investigación, los métodos analizados se agrupan como se aprecia (Tabla 1).

Tabla 1. Clasificación de los métodos de investigación atendiendo a la finalidad investigativa.

\begin{tabular}{|c|c|c|}
\hline \multirow{5}{*}{$\begin{array}{l}\text { Métodos para } \\
\text { la búsqueda de } \\
\text { información }\end{array}$} & La totalidad de los empíricos & $\begin{array}{l}\text { Buscan información directamente en } \\
\text { las unidades de análisis }\end{array}$ \\
\hline & Analítico-sintético & \multirow{4}{*}{$\begin{array}{l}\text { Buscan información mediante } \\
\text { acciones lógicas del pensamiento }\end{array}$} \\
\hline & Histórico-lógico & \\
\hline & Genético & \\
\hline & Sistematización & \\
\hline \multirow{6}{*}{$\begin{array}{l}\text { Métodos para } \\
\text { construcción del } \\
\text { conocimiento }\end{array}$} & Hipotético deductivo & \multirow{3}{*}{ Regularidades externas } \\
\hline & Por analogías & \\
\hline & Sistematización & \\
\hline & Inductivo-Deductivo & \multirow{3}{*}{ Regularidades esenciales } \\
\hline & La modelación & \\
\hline & El sistémico-estructural-funcional & \\
\hline
\end{tabular}

Fuente. Elaboración propia de los autores.

\section{Conclusiones}

I empleo del método dialéctico como metodología general para la obtención del conocimiento científico conduce a clasificar los métodos de investigación en empíricos y racionales, que es otra visión respecto de la clasificación en empíricos y teóricos, comúnmente manejada en los textos de metodología de la investigación científica.

Otro criterio para clasificar los métodos de investigación surge de considerar los diferentes momentos de la lógica interna investigativa, que posibilita agruparlos en métodos para la búsqueda de información y métodos para la construcción de conocimientos, según la función que predomine con mayor frecuencia durante su empleo, lo cual resulta de particular importancia en la etapa de planificación de la investigación, porque facilita que el investigador se oriente adecuadamente en la selección de los métodos por emplear. 


\section{Referencias}

Abbagnano, N. (1963). Storia della filosofía, Torino: UTET.

Álvarez de Zayas, C. (1993). ¿Cómo se modela la investigación científica? La Habana: MES.

Antillón, R. (2002). ¿Cómo lo hacemos? Para construir conocimientos a través de la sistematización de la práctica social. Guadalajara. México: Instituto Mexicano para el Desarrollo Comunitario.

Ardón Mejía, M. (2000). Guía metodológica para la sistematización participativa de experiencias en agricultura sostenible. San Salvador: Programa para la Agricultura Sostenible en Laderas de América Central.

Behar Rivero, D. S. (2008). Introducción a la metodología de la investigación. Shalom.

Bernaldo de Quirós, M. L. y Rodríguez, M. del P. (2004). La sistematización como forma de producción de conocimiento científico, desde una perspectiva no positivista. Revista Confluencia, 1(4). Recuperado de http://bdigital.uncu.edu.ar/327

Bisquera Alzina, R. (1989). Métodos de investigación educativa: guía práctica. Barcelona: CEAC.

Cachón Guillén, V. (2013). Las analogías en la formulación de la teoría electromagnética de la luz de Maxwell. Claves del Pensamiento, 7(14), 11-33. Recuperado de http://www.scielo.org.mx/ scielo.php? script $=$ sci_arttext\&pid $=$ S1870-879X2013 000200001\&lng=es\&tlng=es
Castro Acosta, Y. (2010). Propuesta de procedimientos para la determinación y evaluación de los costos ecológicos: un caso práctico. Observatorio Iberoamericano del Desarrollo Local y la Economía Social, 4(9). Recuperado de http://www.eumed. net/rev/oidles/09/yca.pdf

Cerezal, J. y Fiallo, J. (2005). Cómo investigar en pedagogía. La Habana: Pueblo y Educación.

Charmaz, K. (2006). Constructing Grounded Theory, a practical guide through Qualitative Analysis. London, SAGE Publications.

Dávila Newman, G. (2006). El razonamiento inductivo y deductivo dentro del proceso investigativo en ciencias experimentales y sociales. Laurus, 12, 180-205.

Davis, J., Eisenhardt, K. y Bingham, C. (2007). Complexity theory, market dynamism, and the strategy of simple rules. Stanford, CA: Stanford University.

De la Uz Herrera, M. del C., De la Uz Herrera, M. E., Lemus Sarracino, A., Valdés Santiesteban, M. N. y Padrón Novales, C. I. (2010). Competencia comunicativa en los estudiantes de medicina: diagnóstico preliminar. Revista de Ciencias Médicas de Pinar del Río, 14(1), 314-326.

Dubin, R. (1976). Theory building in applied areas. En M. Dunnette (ed.), Handbook of industrial and organizational psychology (pp. 17-40). Chicago: Rand McNally. 
Fraga Rodríguez, R. y Herrera Padrón, C. (1999). Metodología de la investigación educativa. La Habana.

Gallo, J. y González, E. (2002). Introducción a la metodología de investigación pedagógica y técnica. La Habana.

González, O. (2008). Multimedia educativa para apoyar el proceso de enseñanzaaprendizaje en la asignatura comunicación organizacional. Centro de Información y Gestión Tecnológica de Santiago de Cuba.

HerreraSalazar,M.O.,HerreraSalazar, R.yPérez Martín, F. (2012). Sitio web para el proceso enseñanza-aprendizaje en bioquímica de tecnología de la salud. Edumecentro, 4(2), 125-136. Recuperado de http:// scielo.sld.cu/scielo.php?pid=S2077$28742012000200016 \&$ script $=$ sci arttext\&tlng=pt

Jara, O. (1998). Sistematización de experiencias: búsquedas recientes. Dimensión Educativa, 44.

Jivkova Semova, D. (2011). RTVE sin publicidad: un modelo de financiación en estado de emergencia. Vivat Academia, 116, 75-91. Recuperado de http:// vivatacademia.net/index.php/vivat/ article/view/155

Labarca, A. (2001). Métodos de investigación en educación: un curso modular. Santiago de Chile: Universidad Metropolitana de Ciencias de la Educación.

Lanuez Bayolo, M. del C., Martínez LLantada, M. y Pérez Fernández, V. (s. f.). El maestro y la investigación educativa en el siglo XXI. Recuperado de https://es.scribd. com/doc/55928029/El-Maestro-y-laInvestigacion-Educativa-en-el-Siglo-XXI
León-Barúa, R. (1999). Del diagnóstico a la investigación en medicina. Diagnóstico, 38(6), 293-6. Recuperado de http:// www.fihu-diagnostico.org.pe/revista/ numeros/1998-99/novdic99/293-296.html

Lima Cedro, W. y De Moura, M. O. (2010). Experimento didáctico: un camino metodológico para la investigación en la educación matemática. Unión: Revista Iberoamericana de Educación Matemática, 22, 53-63.

Martínez, Y. (2016). Estándares para eliminar ataques de seguridad en los servicios web. Recuperado de http://publicaciones.uci. cu/index.php/SC/article/viewFile/965/593

Morin, E. (2002). Epistemología de la complejidad. En Nuevos paradigmas, cultura y subjetividad. Buenos Aires: Paidós.

Nocedo de León, I. (1984). Metodología de la investigación pedagógica y psicológica. La Habana: Pueblo y Educación.

Notario de la Torre, Á. (1999). Apuntes para un compendio sobre metodología de la investigación científica. Pinar del Río, Cuba: Universidad de Pinar del Río.

Ordaz-Hernández, A., Chuy-Rodríguez, T. J. y García-Gutiérrez, J. A. (2011). Microzonación sísmica local. Caso de estudio: pueblo deSan Cristóbal, Pinar del Río, Cuba. Avances, 13(3). Recuperado de http:// www.ciget.pinar.cu/Revista/No.2011-3/ art\%EDculos/Microzonaci\%F3n_sismica. pdf

Ortiz Torres, E. A. (2015). La dialéctica en las investigaciones educativas. Manta, Manabí, Ecuador: Mar Abierto. 
Pérez, A. (2014a). El grado de coherencia en sistemas y procesos como criterios de constatación de los resultados científicos. La Habana: Educación Cubana.

Pérez, A. (2014b). Una alternativa para los diseños experimentales. La Habana: Educación Cubana.

Pérez Rodríguez, G., García Batista, G., Nocedo de León, I. y García Inza, M. L. (2009). Metodología de la investigación educacional. La Habana: Pueblo y Educación.

Piaget, J. (1968). El estructuralismo. Buenos Aires: Proteo.

Ramírez, I. (2008). Metodología de la investigación educativa, un acercamiento desde la perspectiva del maestro. Lima: San Marcos.

Reyes Piña, O. L. y Bringas Linares, J. A. (2006). La modelación teórica como método de la investigación científica. Revista Varona, 42. Recuperado de http://www.redalyc. org/html/3606/360635561003/

Rodríguez del Castillo, M. A. (s. f.). La sistematización como resultado científico de la investigación educativa: ¿sistematizar la sistematización?

Rosales Echarri, V. (2002). La metodología de la investigación educativa para la formación del profesional. La Habana.
Suárez Lugo, N. (2014). Tabaco o salud: una decisión social. Horizonte Sanitario, 3(2). Recuperado de http://www.revistas.ujat. $\mathrm{mx} /$ index.php/horizonte/article/view/457

Sutton, R. y Staw, B. (1995). What theory is not. Administrative Science Quarterly, 40, 371-384.

Valle, A. (2007). Algunos modelos importantes en la investigación pedagógica. La Habana: Instituto Central de Ciencias Pedagógicas.

Valledor Estevill, R. y Ceballo Rosales, M. (2005). Temas de metodología de la investigación educacional. Las Tunas: Instituto Superior Pedagógico Pepito Tey.

Véliz Martínez, P. L. y Jorna Calixto, A. R. (2014). Evolución histórica y perspectivas futuras de la medicina intensiva y emergencia como especialidad en Cuba. Educación Médica Superior, 28(3), 592-602. Recuperado de http://scielo.sld.cu/scielo.php?pid=S0864$21412014000300018 \&$ script $=$ sci $_{-}$ arttext\&tlng=en

Whetten, D. A. (1989). What constitutes a theoretical contribution? Academy of Management Review, 14(4), 490-495.

Zúñiga, R. (1992). Sobre el sistematizar. Revista de Trabajo Social, 61, 19-29. Recuperado de https://www.webdepot. umontreal.ca/Usagers/zunigar/MonDepot Public/ Textos\%20en\%20caste-llano/ 9.\%20 Sobre\%20el\%20sistematizar.pdf 